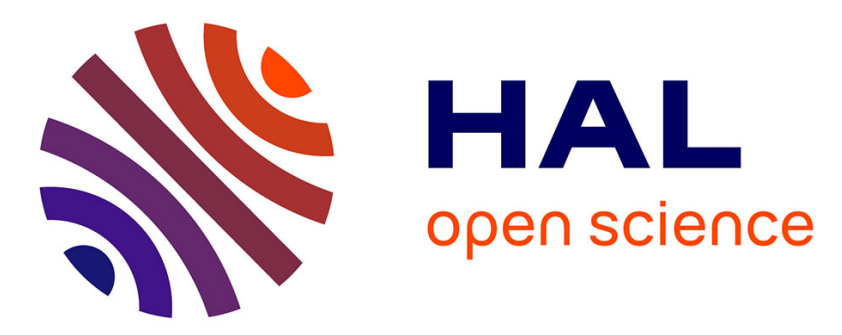

\title{
Observer based actuator fault tolerant control for nonlinear Takagi-Sugeno systems : an LMI approach
}

Dalil Ichalal, Benoît Marx, José Ragot, Didier Maquin

\section{To cite this version:}

Dalil Ichalal, Benoît Marx, José Ragot, Didier Maquin. Observer based actuator fault tolerant control for nonlinear Takagi-Sugeno systems: an LMI approach. 18th Mediterranean Conference on Control and Automation, MED'10, Jun 2010, Marrakech, Morocco. pp.CDROM. hal-00497790

\section{HAL Id: hal-00497790 \\ https://hal.science/hal-00497790}

Submitted on 9 Apr 2014

HAL is a multi-disciplinary open access archive for the deposit and dissemination of scientific research documents, whether they are published or not. The documents may come from teaching and research institutions in France or abroad, or from public or private research centers.
L'archive ouverte pluridisciplinaire HAL, est destinée au dépôt et à la diffusion de documents scientifiques de niveau recherche, publiés ou non, émanant des établissements d'enseignement et de recherche français ou étrangers, des laboratoires publics ou privés. 


\title{
Observer based actuator fault tolerant control for nonlinear Takagi-Sugeno systems : an LMI approach
}

\author{
Dalil Ichalal, Benoit Marx, José Ragot, Didier Maquin
}

\begin{abstract}
A new actuator fault tolerant control strategy is proposed in this paper for nonlinear Takagi-Sugeno (T-S) systems. The control law aims to compensate the actuator faults and allows the system states to track a reference states corresponding to the output of the system in the fault free situation. The design of such a control law requires the knowledge of the faults, this task is achieved with a proportional integral observer (PIO). The robust stability of the system with the fault tolerant control law is analyzed with Lyapunov theory and $\mathcal{L}_{2}$ optimization. Sufficient stability conditions are obtained in terms of linear matrix inequalities (LMIs). The gains of the FTC are obtained by solving these LMIs. A simulation example is finally proposed.
\end{abstract}

Index Terms-Takagi-Sugeno systems, state and fault estimation, PI observer, Lyapunov stability analysis, linear matrix inequality.

\section{INTRODUCTION}

It is well known that the classical control strategies cannot take into account faults affecting a system. Then, if a fault occurs in any component of the system, the stability and the performances of the system cannot be ensured with such control laws. These last years, the problem of fault tolerant control design has been treated and many significant results have been proposed in [14], [2], [16], [17]. These works follow two different ideas. The first one, called passive FTC, considers possible fault situations and take them into account in the step of control design which is similar to the robust control design. It is pointed out in many works that this strategy is usually restrictive. The second approach is the active FTC, which requires a fault diagnosis block providing on line informations on fault detection, isolation and estimation. The reconfigurable control block uses these informations in order to deal with unforeseen faults, to maintain the system stability and to provide an acceptable system trajectory even in faulty situations.

The active fault tolerant control has been developed essentially for linear systems [6], [19], [17], [14] and descriptor linear systems [12]. Clearly, linear models do not often represent accurately physical systems due to the presence of nonlinear behavior. A new representation that combines simplicity and accuracy of nonlinear behaviors was introduced, initially, in [20] and known under the name TakagiSugeno (T-S) models. The idea is to consider a set of linear sub-systems. An interpolation of all these sub-models

\footnotetext{
All the authors are with the Centre de Recherche en Automatique de Nancy (CRAN), Nancy-Université, 2, avenue de la forêt de Haye 54516 Vandoeuvre-les-Nancy \{dalil.ichalal, benoit.marx, jose.ragot, didier.maquin\}eensem. inpl-nancy. fr
}

with nonlinear functions satisfying the sum convex property allows to obtain the global behavior of the system described in a large operating range. One can cite some works in the FTC field for nonlinear systems, for example, in [5], the authors gave a method for actuator faults by using their estimations, for nonlinear descriptor systems with Lipschitz nonlinearities. In [18], a method which requires only the fault isolation was proposed for T-S systems. It was based on a bank of observer based controllers. A switching mechanism is then designed depending on the obtained residuals. More recently, Witczak proposed in [23] an FTC strategy based on a reference model for open-loop T-S systems.

This paper is dedicated to the design of a fault tolerant control strategy for nonlinear systems described by TakagiSugeno models. This approach is an extension, the work proposed in [23], to T-S systems where the weighting functions of the T-S system are affected by faults. Thus, the premise variables of the reference model are not the same as those of the faulty system. The main idea is to re-use the nominal control input developed in fault-free case for which two terms, related to the occurred fault and the tracking error trajectory between the system and a reference model, are added. The reference trajectory is provided from a reference model representing the system without faults. In addition, the control law requires the knowledge of the state of the system and the faults affecting it. For that purpose, a PI observer is used to estimate simultaneously these signals.

\section{A. Takagi-Sugeno structure for modeling}

Let us consider a nonlinear system described by a T-S structure

$$
\left\{\begin{array}{l}
\dot{x}(t)=\sum_{i=1}^{r} \mu_{i}(\xi(t))\left(A_{i} x(t)+B_{i} u(t)\right) \\
y(t)=\sum_{i=1}^{r} \mu_{i}(\xi(t)) C_{i} x(t)
\end{array}\right.
$$

where $x(t) \in \mathbb{R}^{n}$ is the state vector, $u(t) \in \mathbb{R}^{m}$ is the input vector, and $y(t) \in \mathbb{R}^{p}$ represents the output vector. $A_{i} \in$ $\mathbb{R}^{n \times n}, B_{i} \in \mathbb{R}^{n \times m}, C_{i} \in \mathbb{R}^{p \times n}$ and $D_{i} \in \mathbb{R}^{p \times m}$ are known matrices. The functions $\mu_{i}(\xi(t))$ are the weighting functions depending on the variables $\xi(t)$ which can be measurable (as the input or the output of the system) or non measurable variables (as the state of the system). These functions verify the following properties

$$
\left\{\begin{array}{l}
\sum_{i=1}^{r} \mu_{i}(\xi(t))=1 \\
0 \leq \mu_{i}(\xi(t)) \leq 1 \quad \forall i \in\{1,2, \ldots, r\}
\end{array}\right.
$$


Obtaining a T-S model (1) can be performed from different methods such as linearization of a nonlinear model around some operating points and using adequate weighting functions. It can be also obtained by black-box approaches which allow to identify the parameters of the model from input-output data. Finally, an interesting approach to obtain a model in the form (1) is the well-known nonlinear sector transformations [21], [15]. Indeed, this transformation allows to obtain an exact T-S representation of a general nonlinear model with no information loss, in a compact state space.

Thanks to the convex sum property of the weighing functions (2), it is possible to generalize some tools developed in the linear domain to the nonlinear systems. This representation is very interesting in the sense that it simplifies the stability study of nonlinear systems and the design of control laws and observers. In [3], [7], [10], the stability and stabilization tools are inspired from the study of linear systems. In [1], [13], the authors worked on the problem of state estimation and diagnosis of T-S fuzzy systems. The proposed approaches in these last papers rely on the generalization of the classical observers (Luenberger Observer [11] and Unknown Input Observer (UIO) [4]) to the nonlinear domain.

\section{B. Notations and preliminaries}

Let us consider the matrix $Y_{i j}$ with appropriate dimension, and $\mu_{i}($.$) nonlinear functions satisfying the convex sum$ property. The following notation is defined

$$
Y_{\xi \xi}=\sum_{i=1}^{r} \sum_{j=1}^{r} \mu_{i}(\xi(t)) \mu_{j}(\xi(t)) Y_{i j}
$$

Lemma 1: ([22]) The inequality

$$
\sum_{i=1}^{r} \sum_{j=1}^{r} \mu_{i}(\xi(t)) \mu_{j}(\xi(t)) Y_{i j}<0
$$

holds if

$$
\begin{aligned}
Y_{i i} & <0, i=1, \ldots, r \\
\frac{2}{r-1} Y_{i i}+Y_{i j}+Y_{j i} & <0, i, j=1, \ldots, r, i \neq j
\end{aligned}
$$

Lemma 2: (Congruence) Let two matrices $P$ and $Q$, if $P$ is positive definite and if $Q$ is a full column rank matrix, than the matrix $Q P Q^{T}$ is positive definite.

Notation 1: For any square matrix $M, \mathbb{S}(M)$ is defined by :

$$
\mathbb{S}=M+M^{T}
$$

\section{FAULT TOLERANT CONTROL OF T-S FUZZY SYSTEMS}

\section{A. FTC strategy}

Let us consider the T-S reference model without faults described by (1). The faulty system is given by

$$
\left\{\begin{array}{l}
\dot{x}_{f}(t)=\sum_{i=1}^{r} \mu_{i}\left(\xi_{f}(t)\right)\left(A_{i} x_{f}(t)+B_{i}\left(u_{f}(t)+f(t)\right)\right) \\
y_{f}(t)=\sum_{i=1}^{r} \mu_{i}(\xi(t)) C_{i} x_{f}(t)
\end{array}\right.
$$

Note that, the weighting functions depend on a faulty premise variable $\xi_{f}(t)$. Indeed, if these last are the input of the system, which can depend on the state $x_{f}(t)$ in closedloop, or the output $y_{f}(t)$, necessarily the fault affects these variables.

The goal is to design the control law $u_{f}(t)$ such that the system state $x_{f}(t)$ converges toward the reference state $x(t)$ given by the reference model (1). The control strategy is illustrated in the figure 1.

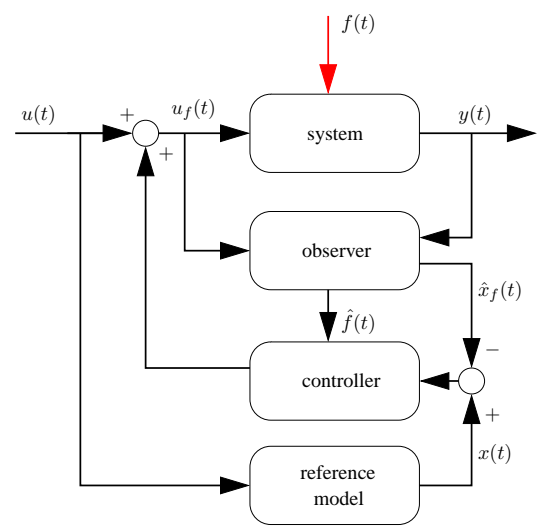

Fig. 1. Fault tolerant control scheme

We propose the following structure for the control law

$$
u_{f}(t)=-\hat{f}(t)+K\left(x(t)-\hat{x}_{f}(t)\right)+u(t)
$$

The matrices $K$ is determined in order to ensure the stability of the system even if faults occur and to minimize the state error between $x_{f}(t)$ and $x(t)$. By analyzing the structure of $u_{f}(t)$ given in equation (9), the estimation of the state vector $x_{f}(t)$ and faults $f(t)$ are required. This task is performed via a Proportional-Integral observer simultaneously estimating the state and the faults of the system.

Let us consider the PI observer

$$
\begin{aligned}
\dot{\hat{x}}_{f}(t) & =\sum_{i=1}^{r} \mu_{i}\left(\xi_{f}(t)\right)\left(A_{i} \hat{x}_{f}(t)+B_{i}\left(u_{f}(t)+\hat{f}(t)\right)\right. \\
& \left.+H_{1 i}\left(y_{f}(t)-\hat{y}_{f}(t)\right)\right) \\
\dot{\hat{f}}(t) & =\sum_{i=1}^{r} \mu_{i}\left(\xi_{f}(t)\right)\left(H_{2 i}\left(y_{f}(t)-\hat{y}_{f}(t)\right)\right) \\
\hat{y}_{f}(t) & =\sum_{i=1}^{r} \mu_{i}\left(\xi_{f}(t)\right) C_{i} \hat{x}_{f}(t)
\end{aligned}
$$

In fact if $\xi_{f}(t)$ is assumed to be known, the observer weighting functions depend on the same premise variable as the system (8).

The output error between the system (8) and the observer (10) is written by

$$
y_{f}(t)-\hat{y}_{f}(t)=\sum_{i=1}^{r} \mu_{i}\left(\xi_{f}(t)\right) \tilde{C}_{i} e_{a}(t)
$$

where

$$
\tilde{C}_{i}=\left[\begin{array}{ll}
C_{i} & 0
\end{array}\right]
$$




$$
e_{a}(t)=x_{a}(t)-\hat{x}_{a}(t), \quad x_{a}(t)=\left[\begin{array}{c}
x_{f}(t) \\
f(t)
\end{array}\right]
$$

The dynamic of the trajectory tracking error $e(t)=x(t)-x_{f}(t)$, obeys to the differential equation

$$
\begin{aligned}
\dot{e}(t) & =\sum_{i=1}^{r} \mu_{i}(\xi(t))\left(A_{i} x(t)+B_{i} u(t)\right) \\
& -\mu_{i}\left(\xi_{f}(t)\right)\left(A_{i} x_{f}(t)+B_{i}\left(u_{f}(t)-f(t)\right)\right) \\
\dot{e}(t) & =\sum_{i=1}^{r} \mu_{i}\left(\xi_{f}(t)\right)\left(A_{i} e(t)-B_{i}(f(t)+\hat{f}(t))\right. \\
& \left.-B_{i} K\left(x_{f}(t)-\hat{x}_{f}(t)\right)\right)+\delta(t) \\
& =\sum_{i=1}^{r} \mu_{i}\left(\xi_{f}(t)\right)\left(\left(A_{i}-B_{i} K\right) e(t)\right. \\
& \left.-\tilde{L}_{i} e_{a}(t)\right)+\delta(t)
\end{aligned}
$$

where

$$
\begin{gathered}
\tilde{L}_{i}=\left(\begin{array}{cc}
B_{i} K & B_{i}
\end{array}\right), \quad e_{a}=x_{a}(t)-\hat{x}_{a}(t) \\
\delta(t)=\sum_{i=1}^{r}\left(\mu_{i}(\xi(t))-\mu_{i}\left(\xi_{f}(t)\right)\right)\left(A_{i} x(t)+B_{i} u(t)\right)
\end{gathered}
$$

Assume that $\dot{f}(t)=0$, the system (8) can be written in augmented form

$$
\left\{\begin{array}{l}
\dot{x}_{a}(t)=\sum_{i=1}^{r} \mu_{i}\left(\xi_{f}(t)\right)\left(\tilde{A}_{i} x_{a}(t)+\tilde{B}_{i} u_{f}(t)\right) \\
y_{f}(t)=\sum_{i=1}^{r} \mu_{i}\left(\xi_{f}(t)\right) \tilde{C}_{i} x_{a}(t)
\end{array}\right.
$$

where

$$
\tilde{A}_{i}=\left(\begin{array}{cc}
A_{i} & B_{i} \\
0 & 0
\end{array}\right), \quad \tilde{B}_{i}=\left(\begin{array}{c}
B_{i} \\
0
\end{array}\right),
$$

The pairs $\left(\tilde{A}_{i}, \tilde{C}_{j}\right), i, j=1, \ldots, r$ are assumed to be observable (or at least detectable). The state and fault estimation error $e_{a}(t)=x_{a}(t)-\hat{x}_{a}(t)$ between the system (20) and the observer (10)-(11) evolves following the equation

$$
\dot{e}_{a}(t)=\sum_{i=1}^{r} \sum_{j=1}^{r} \mu_{i}\left(\xi_{f}(t)\right) \mu_{j}\left(\xi_{f}(t)\right)\left(\left(\tilde{A}_{i}-H_{i} \tilde{C}_{j}\right) e_{a}(t)\right)
$$

The concatenation of the state tracking trajectory error and the state and faults estimation errors allows to write, from (16) and (22), a new augmented system written by

$$
\dot{\tilde{e}}(t)=\sum_{i=1}^{r} \sum_{j=1}^{r} \mu_{i}\left(\xi_{f}(t)\right) \mu_{j}\left(\xi_{f}(t)\right) \tilde{A}_{i j} \tilde{e}(t)+\tilde{\Gamma} \delta(t)
$$

where

$$
\begin{gathered}
\tilde{e}(t)=\left(\begin{array}{c}
x(t)-x_{f}(t) \\
x_{a}(t)-\hat{x}_{a}(t)
\end{array}\right), \quad \tilde{\Gamma}=\left(\begin{array}{c}
I_{n} \\
0
\end{array}\right) \\
\tilde{A}_{i j}=\left(\begin{array}{cc}
A_{i}-B_{i} K & -\tilde{L}_{i} \\
0 & \tilde{A}_{i}-H_{i} \tilde{C}_{j}
\end{array}\right)
\end{gathered}
$$

Remark 1: One can note that in the previous section, the weighting functions depend on the premise variable $\xi_{f}(t)$.
It can be external known variable which is not affected by faults. Indeed, in [23], the authors proposed a method for this case with application to the three tank system in openloop control. In this case, $\xi(t)=\xi_{f}(t)$ and the equation (23) becomes

$$
\dot{\tilde{e}}(t)=\sum_{i=1}^{r} \sum_{j=1}^{r} \mu_{i}(\xi(t)) \mu_{j}(\xi(t)) \tilde{A}_{i j} \tilde{e}(t)
$$

In Takagi-Sugeno modeling, it is often considered that the premise variable $\xi(t)$ is the input, the output or the state of the system, which are necessarily affected by faults. Consequently, $\xi(t) \neq \xi_{f}(t)$. In addition if $\xi_{f}(t)$ is measurable the state estimation error and the state tracking error are expressed by (23). Now, with this considerations, when $\xi(t)=u(t)$ and $\xi_{f}(t)=u_{f}(t)$, the term $\delta(t)$ does not converge to zero if $x_{f}(t)$ converges to the reference state $x(t)$ but if $\xi(t)=y(t)$ and $\xi_{f}(t)=y_{f}(t)$, the tolerant control allows the convergence of $x_{f}(t)$ to $x(t)$ and $y_{f}(t)$ to $y(t)$, then the term $\delta(t)$ converges also to zero which gives better results compared to the case where $\xi(t)=u(t)$. The same problem can appear if the output is also affected by faults. In these cases, the fault tolerant control design aims to minimize the difference between $x_{f}(t)$ and $x(t)$ and to minimize the $\mathcal{L}_{2}$ gain of the transfer from $\delta(t)$ to the state tracking error.

\section{B. Fault tolerant control design}

The gains $K, H_{1 i}$ and $H_{2 i}$ are determined by solving the optimization problem under LMI constraints given in theorem 1.

Theorem 1: Let be $\mu$ a positive scalar. The system (23) that generates the state tracking error $e(t)$ and the state and fault estimation errors $e_{a}(t)$ is stable and the $\mathcal{L}_{2}$-gain of the transfer from $\delta(t)$ to $e_{a}(t)$ is bounded if there exists symmetric and positive definite matrices $X_{1}, X_{2}, P_{2}$ and $P_{3}$, matrices $\bar{H}_{i}$ and $\bar{K}$ and positive scalars $\bar{\gamma}$ solution to the following optimization problem

$$
\min _{X_{1}, X_{2}, P_{2}, \bar{K}_{i}, \bar{H}_{i},} \quad \bar{\gamma} \text { s.t. } \quad(5)-(6)
$$

where

$$
\begin{gathered}
Y_{i j}=\left(\begin{array}{ccccc}
\Psi_{i} & -B_{i} M & 0 & I_{n} & X_{1} \\
* & -2 \mu X & \mu I & 0 & 0 \\
* & * & \Delta_{i j} & 0 & 0 \\
* & * & * & -\bar{\gamma} I_{n} & 0 \\
* & * & * & * & -I_{n}
\end{array}\right)<0 \\
\Psi_{i}=A_{i} X_{1}+X_{1} A_{i}^{T}-B_{i} \bar{K}-\bar{K}^{T} B_{i}^{T} \\
\Delta_{i j}= \\
P_{2} \tilde{A}_{i}+\tilde{A}_{i}^{T} P_{2}-\bar{H}_{i} \tilde{C}_{j}-\tilde{C}_{j}^{T} \bar{H}_{i}^{T} \\
M=\left(\begin{array}{cc}
\bar{K} & X_{2}
\end{array}\right) \\
X=\left(\begin{array}{cc}
X_{1} & 0 \\
0 & X_{2}
\end{array}\right)
\end{gathered}
$$

The controller gains and those of the observer are computed from

$$
\begin{aligned}
H_{i} & =\left(\begin{array}{c}
H_{1 i} \\
H_{2 i}
\end{array}\right)=P_{2}^{-1} \bar{H}_{i} \\
K & =\bar{K} X_{1}^{-1}
\end{aligned}
$$


and the attenuation level of the transfer from $\delta(t)$ to $e(t)$ is obtained by

$$
\gamma=\sqrt{\bar{\gamma}}
$$

Proof: The gains $H_{i}$ and $K$ are obtained by stability analysis of the system described by the differential equation (23) by using Lyapunov theory with a quadratic function.

Let us chose the following quadratic Lyapunov function

$$
V(\tilde{e}(t))=\tilde{e}(t)^{T} P \tilde{e}(t), \quad P=P^{T}>0
$$

where $P$ is chosen as follows

$$
P=\left(\begin{array}{cc}
P_{1} & 0 \\
0 & P_{2}
\end{array}\right)
$$

The time derivative of the function $V(\tilde{e}(t))$ is given by

$$
\begin{aligned}
\dot{V}(\tilde{e}(t)) & =\sum_{i=1}^{r} \sum_{j=1}^{r} \mu_{i}\left(\xi_{f}(t)\right) \mu_{j}\left(\xi_{f}(t)\right) \tilde{e}(t)^{T} \mathcal{M}_{i j} \tilde{e}(t) \\
& +2 \tilde{e}(t)^{T} P \tilde{\Gamma} \delta(t)
\end{aligned}
$$

where

$$
\mathcal{M}_{i j}=\mathbb{S}\left(\left(\begin{array}{cc}
\Lambda_{i} & -P_{1} \tilde{L}_{i} \\
0 & P_{2} \tilde{A}_{i}-P_{2} H_{i} \tilde{C}_{j}
\end{array}\right)\right)
$$

where

$$
\Lambda_{i}=P_{1} A_{i}-P_{1} B_{i} K
$$

and $\mathbb{S}$ is a function defined in the notation 1 .

In addition, the term $\delta(t)$ depends on $x(t), u(t)$ which are bounded, then it is also bounded. So, the objective is to minimize the $\mathcal{L}_{2}$-gain of the transfer from $\delta(t)$ to the state tracking error $e(t)$, this is formulated by

$$
\frac{\|e(t)\|_{2}}{\|\delta(t)\|_{2}}<\gamma, \quad\|\delta(t)\|_{2} \neq 0
$$

Then, we are seeking to ensure asymptotic convergence toward zero if $\delta(t)=0$ and to guarantee a bounded $\mathcal{L}_{2}$-gain if $\delta(t) \neq 0$. This problem can be formulated as follows

$$
\dot{V}(\tilde{e}(t))+e(t)^{T} e(t)-\gamma^{2} \delta(t)^{T} \delta(t)<0
$$

After some calculation, the inequality (42) is negative if the following conditions hold

$$
N_{\xi \xi}=\sum_{i=1}^{r} \sum_{j=1}^{r} \mu_{i}\left(\xi_{f}(t)\right) \mu_{j}\left(\xi_{f}(t)\right) \mathcal{N}_{i j}<0
$$

where

$$
\mathcal{N}_{i j}=\left(\begin{array}{ccc}
\mathbb{S}\left(\Lambda_{i}\right)+I_{n} & -P_{1} L_{i} & P_{1} \\
0 & \mathbb{S}\left(P_{2} \tilde{A}_{i}-P_{2} H_{i} \tilde{C}_{j}\right) & 0 \\
P_{1} & 0 & -\gamma^{2} I
\end{array}\right)
$$

with the congruence lemma, we obtain

$$
N_{\xi \xi}<0 \Leftrightarrow W N_{\xi \xi} W^{T}<0
$$

where

$$
W=\left(\begin{array}{ccc}
P_{1}^{-1} & 0 & 0 \\
0 & X & 0 \\
0 & 0 & I
\end{array}\right), X=\left(\begin{array}{cc}
P_{1}^{-1} & 0 \\
0 & X_{2}
\end{array}\right)
$$

$X_{2}$ is symetric and positive definite matrix. The following is then obtained

$$
\sum_{i=1}^{r} \sum_{j=1}^{r} \mu_{i}\left(\xi_{f}(t)\right) \mu_{j}\left(\xi_{f}(t)\right)\left(\begin{array}{ccc}
\Xi_{i} & -L_{i} X & I_{n} \\
* & X \Delta_{i j} X & 0 \\
* & * & -\gamma^{2} I
\end{array}\right)<0
$$

where

$$
\begin{aligned}
\Xi_{i} & =A_{i} P_{1}^{-1}+P_{1}^{-1} A_{i}^{T}-B_{i} K P_{1}^{-1} \\
& -P_{1}^{-1} K^{T} B_{i}^{T}+P_{1}^{-1} P_{1}^{-1} \\
\Delta_{i j} & =P_{2} \tilde{A}_{i}+\tilde{A}_{i}^{T} P_{2}-P_{2} H_{i} \tilde{C}_{j}-\tilde{C}_{j}^{T} H_{i}^{T} P_{2}
\end{aligned}
$$

The negativity of (47) imposes the negativity of $\Delta_{i j}$ which allows to use the following property

$$
\begin{aligned}
& \left(X+\mu \Delta_{i j}^{-1}\right)^{T} \Delta_{i j}\left(X+\mu \Delta_{i j}^{-1}\right) \leq 0 \\
\Leftrightarrow \quad & X \Delta_{i j} X \leq-\mu\left(X+X^{T}\right)-\mu^{2} \Delta_{i j}^{-1}
\end{aligned}
$$

(47) can then be bounded in the following way

$$
Y_{\xi \xi}=\sum_{i=1}^{r} \sum_{j=1}^{r} \mu_{i}\left(\xi_{f}(t)\right) \mu_{j}\left(\xi_{f}(t)\right) Y_{i j}<0
$$

where

$$
Y_{i j}=\left(\begin{array}{cccc}
\Xi_{i} & -L_{i} X & 0 & I_{n} \\
* & -2 \mu X & \mu I & 0 \\
* & * & \Delta_{i j} & 0 \\
* & * & * & -\gamma^{2} I
\end{array}\right)
$$

After the use of the lemma 1, in order to express the inequalities in linear form with respect to $P_{1}^{-1}, P_{2}, K$, and $H_{i}$, the following change of variables are used

$$
X_{1}=P_{1}^{-1}, \quad \bar{K}=K X_{1}, \quad \bar{H}_{i}=P_{2} H_{i}, \quad \bar{\gamma}=\gamma^{2}
$$

In addition

$$
\tilde{L}_{i} X=B_{i}\left(\begin{array}{ll}
K & I
\end{array}\right) X=B_{i}\left(\begin{array}{cc}
\bar{K} & X_{2}
\end{array}\right)
$$

Then, the relaxed stability conditions satisfying the attenuation level of the $\mathcal{L}_{2}$ gain of the transfer from $\delta(t)$ to the state tracking error $e(t)$, given in theorem 1 , are obtained.

Remark 2: The assumption that the fault signal is constant over the time is restrictive, but in many practical situations where the faults are slowly time-varying signals, the estimation of the faults is correct, and the proposed FTC scheme can be applied. In the case where the faults are not slowly time-varying or constant, the Proportional Integral Observer (PIO) can be replaced by a Proportional Multiple Integral Observer (PMIO) (see [8]). Such is able to estimate a large class of time-varying signals which satisfies the following assumption

$$
f^{(q+1)}=0
$$

The principle of this observer is based on the estimation of all the $q^{t h}$ derivatives of the signal $f(t)$. This observer can also be extended to the case where $f^{(q+1)}$ is bounded.

\section{Simulation example}

To illustrate the proposed actuator fault tolerant control strategy for T-S systems with measurable premise variables affected by the faults, we proposed two academic examples. 
A. First case : $\xi(t)=u(t)$

Consider a T-S system described by

$$
\left\{\begin{array}{l}
\dot{x}_{f}(t)=\sum_{i=1}^{r} \mu_{i}(u(t))\left(A_{i} x_{f}(t)+B_{i} u_{f}(t)+B_{i} f(t)\right) \\
y_{f}(t)=C x_{f}(t)
\end{array}\right.
$$

where

$$
\begin{gathered}
A_{1}=\left[\begin{array}{ccc}
-2 & 1 & 1 \\
1 & -3 & 0 \\
2 & 1 & -8
\end{array}\right], A_{2}=\left[\begin{array}{ccc}
-3 & 2 & -2 \\
0 & -3 & 0 \\
5 & 2 & -4
\end{array}\right], \\
B_{1}=\left[\begin{array}{l}
0 \\
1 \\
1
\end{array}\right], B_{2}=\left[\begin{array}{l}
1 \\
1 \\
0
\end{array}\right], C=\left[\begin{array}{lll}
1 & 1 & 1 \\
1 & 0 & 1
\end{array}\right]
\end{gathered}
$$

The weighting functions depend on the input $u(t)$ which is the nominal control of the system in the fault-free case ; they are defined by $\mu_{1}(u(t))=(1-u(t)) / 2$ and $\mu_{2}(u(t))=1-$ $\mu_{1}(u(t))$. To apply the proposed FTC strategy, the following reference model is considered

$$
\dot{x}(t)=\sum_{i=1}^{r} \mu_{i}(u(t))\left(A_{i} x(t)+B_{i} u(t)\right), y(t)=C x(t)
$$

The fault $f(t)$ is time varying and defined as follows

$$
f(t)= \begin{cases}-u(t) & t \geq 10 \\ 0 & t<10\end{cases}
$$

To increase the observer performances, a pole assignment is performed in $\{z|\Re(z)<-14| z \mid,<20\}$ in order to enhance the convergence speed of the state estimation errors toward zero and to reduce the oscillatory phenomenon.

Solving the optimization problem under LMI constraints in theorem 1 with $\mu=20$, results in the following matrices

$$
\begin{gathered}
H_{11}=\left[\begin{array}{cc}
-24.84 & 59.47 \\
30.05 & -29.75 \\
31.54 & -43.02
\end{array}\right], H_{12}=\left[\begin{array}{cc}
-11.03 & 45.34 \\
31.58 & -33.25 \\
17.80 & -26.25
\end{array}\right], \\
H_{21}=\left[\begin{array}{ll}
337.82 & -356.67
\end{array}\right], H_{21}=\left[\begin{array}{ll}
338.57 & -353.93
\end{array}\right] \\
K=\left[\begin{array}{lll}
6.5179 & 4.9204 & 1.2659
\end{array}\right], \gamma=0.4721
\end{gathered}
$$

The proportional-integral observer provides the state and fault estimation which errors are depicted in the figures 3 (top) and fault estimation in the figure 2 (top). The figure 3 (bottom) shows the state trajectory tracking errors between the state of the system and those of the reference model with the FTC control law $u_{f}(t)$ depicted in the figure 2 (bottom). Finally, the figure 4 compares, in the one hand, the nominal control input (in fault-free case) and the new control input when fault occurs, and in the other hand the states of the system controlled by the FTC control law, those of the reference model and those of the system with faults but without FTC control law. Even if a fault occurs, the system trajectory follows the trajectory of the reference model which represents the trajectory of the system in the fault-free situation. Thus, the FTC control law compensates the fault and allows a normal functioning of the system in the presence of faults.
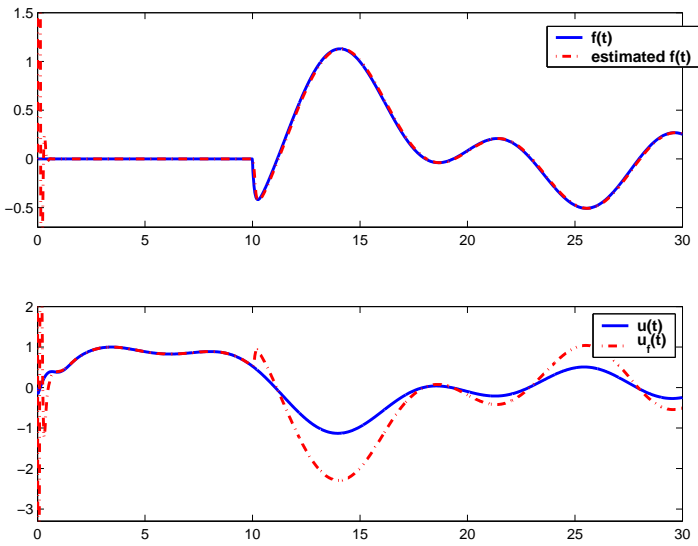

Fig. 2. Fault and its estimates (top) Nominal control and FTC (bottom)
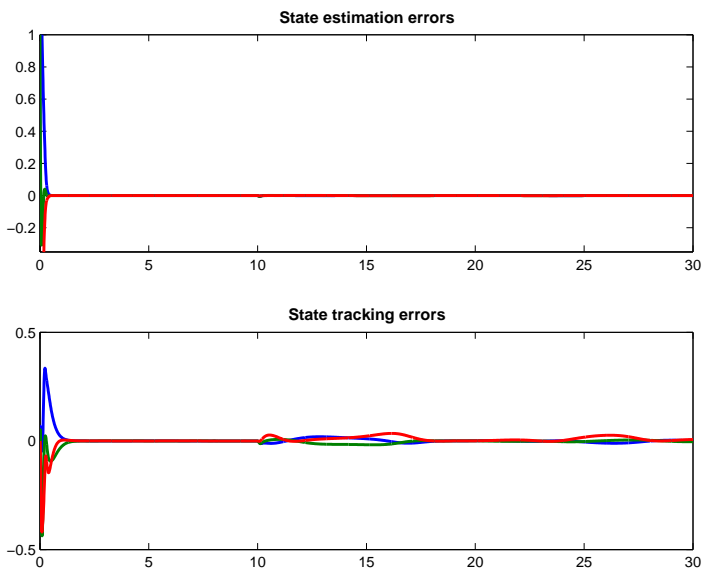

Fig. 3. State estimation errors (top) State tracking errors (bottom)
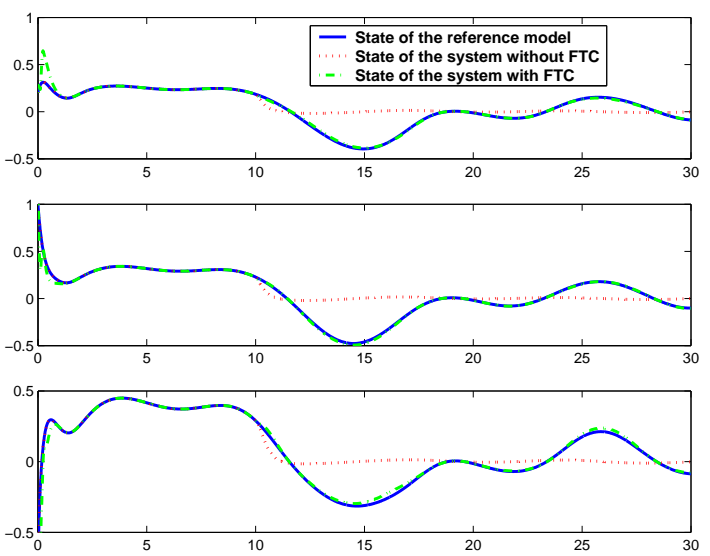

Fig. 4. Comparsion between states of the system without fault, states with fault and nominal control and states with fault and FTC

\section{B. Second case : $\xi(t)=y(t)$}

In this subsection, the previous system is considered, but with weighting functions depending on the first component of the system output vector. The figure 5 illustrates the state estimation errors (top) and the state tracking errors (bottom). It is clear that the use of weighting functions depending on the output of the system provides better results than 
the case where they are depending on the control input. This is due to the fact that the system is only affected by actuator faults and the perturbation term $\delta(t)$ converges to zero when $y_{f}(t)$ converges to the reference $y(t)$. But in the previous simulation, the term $\delta(t)$ did not converge to zero, in the presence of fault, because $u(t) \neq u_{f}(t)$ which leads to $\mu_{i}(u(t)) \neq \mu_{i}\left(u_{f}(t)\right)$. As a conclusion, considering the
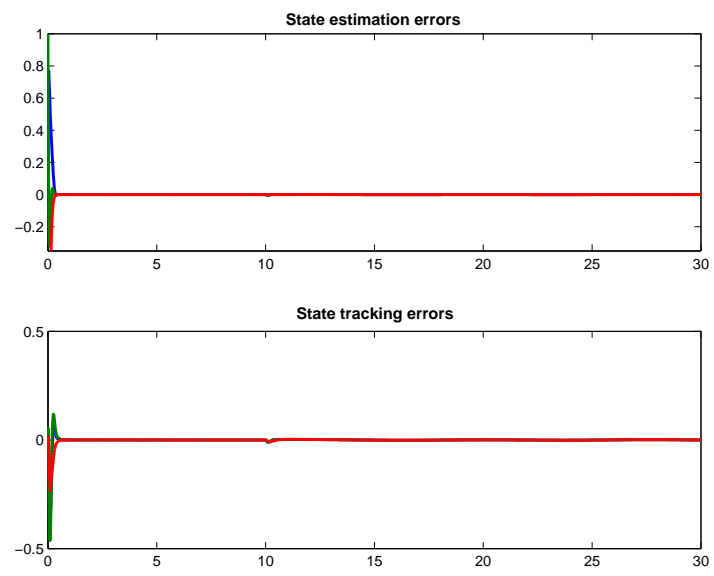

Fig. 5. State estimation errors (top) State tracking errors (bottom)

problem of fault tolerant control of T-S systems with actuator faults, it is more interesting to use the output of the system as a premise variable. However, in the simultaneously occurring actuator and sensor faults, better results are obtained by using the state of the system as a premise variable, this is more difficult and general case but the obtained state error tracking is less than ones obtained above, first results on this point are submitted in [9].

\section{CONCLUSION}

This paper is dedicated to the design of an active fault tolerant control law for nonlinear Takagi-Sugeno fuzzy systems. A reference model is used and the proposed control law is then designed for guaranteeing the convergence of the states of the system to the states of the reference model even if fault occurs. This control law uses the nominal control input developed for the system in fault-free case and two additional terms related to the estimated fault and the trajectory tracking error. The stability is studied with the Lyapunov theory and L2 optimization. The LMI formalism is used in order express stability conditions in term of linear matrix inequalities. Future works will be devoted to the study of the case when the weighting functions depend on unmeasurable variable as the system state. Indeed, the interest of this case is the possibility to deal with simultaneous actuator and sensor faults. In addition, it is interesting to develop the FTC control law by taking into account modeling uncertainties and some external perturbations.

\section{REFERENCES}

[1] A. Akhenak, M. Chadli, J. Ragot, and D. Maquin. Fault detection and isolation using sliding mode observer for uncertain Takagi-Sugeno fuzzy model. In 16th Mediterranean Conference on Control and Automation Congress Centre, Ajaccio, France, June 25-27 2008.
[2] M. Blanke, M. Kinnaert, J. Lunze, and M. Staroswiecki. Diagnosis and Fault-Tolerant Control. Springer, 2006.

[3] M. Chadli, D. Maquin, and J. Ragot. Non quadratic stability analysis of Takagi-Sugeno systems. In IEEE Conference on Decision and Control, CDC'2002, Las Vegas, Nevada, USA, 2002.

[4] M. Darouach, M. Zasadzinski, and S.J. Xu. Full-order observers for linear systems with unknown inputs. IEEE Transactions on Automatic Control, 39(3):606-609, March 1994.

[5] Z. Gao and S. X. Ding. Actuator fault robust estimation and faulttolerant control for a class of nonlinear descriptor systems. Automatica, 43(5):912-920, 2007.

[6] Z. Gao and P.J. PJ Antsaklis. Reconfigurable control system design via perfect model following. International Journal of Control, 56(4)::783798, 1992.

[7] T-M. Guerra, A. Kruszewski, L. Vermeiren, and H. Tirmant. Conditions of output stabilization for nonlinear models in the TakagiSugeno's form. Fuzzy Sets and Systems, 157(9):1248-1259, May 2006.

[8] D. Ichalal, B. Marx, J. Ragot, and D. Maquin. Simultaneous state and unknown inputs estimation with PI and PMI observers for Takagi-Sugeno model with unmeasurable premise variables. In 17th Mediterranean Conference on Control and Automation, MED'09, Thessaloniki, Greece, June 24-26 2009.

[9] D. Ichalal, B. Marx, J. Ragot, and D. Maquin. Fault tolerant control for Takagi-Sugeno systems with unmeasurable premise variables by trajectory tracking. In IEEE International workshop on Industrial electronics, Bari, Italy, 4 - 7 July 2010. (submitted).

[10] A. Kruszewski, R. Wang, and T.M. Guerra. Nonquadratic stabilization conditions for a class of uncertain nonlinear discrete time TS fuzzy models: A new approach. IEEE Transactions on Automatic Control, 53(2):606 - 611, March 2008.

[11] D.G. Luenberger. An introduction to observers. IEEE Transactions on Automatic Control, 16:596-602, 1971.

[12] B. Marx, D. Koenig, and D. Georges. Robust fault tolerant control for descriptor systems. IEEE Transactions on Automatic Control, 49:1869-1875, 2004.

[13] B. Marx, D. Koenig, and J. Ragot. Design of observers for takagi sugeno descriptor systems with unknown inputs and application to fault diagnosis. IET Control Theory and Application, 1:1487-1495, 2007.

[14] M.M. Mufeed, J Jiang, and Z. Zhang. Active Fault Tolerant Control Systems : Stochastic Analysis and Synthesis. Springer, 2003.

[15] A.M. Nagy, G. Mourot, B. Marx, G. Schutz, and J. Ragot. Model structure simplification of a biological reactor. In 15th IFAC Symposium on System Identification, SYSID'09, Saint Malo, France, 2009.

[16] Henrik Niemann and Jakob Stoustrup. Passive fault tolerant control of a double inverted pendulum-a case study. Control Engineering Practice, 13(8):1047-1059, August 2005.

[17] H. Noura, D. Sauter, F. Hamelin, and D. Theilliol. Fault-tolerant control in dynamic systems: Application to a winding machine. IEEE Control Systems Magazine, 20(1):33-49, 2000.

[18] M. Oudghiri. Commande multimodles tolérante aux défauts : application au contrôle de la dynamique d'un véhicule automobile. $\mathrm{PhD}$ thesis, Université de Picardie Jules Verne, Amiens, France, 2008.

[19] M. Staroswiecki. Fault tolerant control : the pseudo-inverse method revisited. In 16th IFAC World Congress, Seoul, Korea, 2005.

[20] T. Takagi and M. Sugeno. Fuzzy identification of systems and its applications to modeling and control. IEEE Transactions on Systems, Man, and Cybernetics, 15:116-132, 1985.

[21] K. Tanaka and H.O. Wang. Fuzzy Control Systems Design and Analysis: A Linear Matrix Inequality Approach. John Wiley and Sons, 2001.

[22] H.D. Tuan, P. Apkarian, T. Narikiyo, and Y. Yamamoto. Parameterized linear matrix inequality techniques in fuzzy control system design. ieee trans. on fuzzy systems. IEEE Transaction on Fuzzy Systems, 9:324332, 2001.

[23] M. Witczak, L. Dziekan, V. Puig, and J. Korbicz. Design of a fault-tolerant control scheme for Takagi-Sugeno fuzzy systems. In 16th Mediterranean Conference on Control and Automation, Ajaccio, France, 25-27 June 2008. 